\title{
Average Consensus in the Presence of Dynamically Changing Directed Topologies and Time Delays
}

Themistoklis Charalambous ${ }^{\star}$ and Christoforos N. Hadjicostis $\sharp$

* Department of Automatic Control, Royal Institute of Technology (KTH) $\sharp$ Department of Electrical and Computer Engineering, University of Cyprus
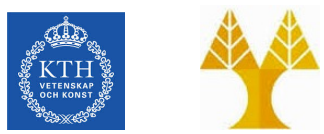

IEEE Conference on Decision and Control (CDC '14)

Los Angeles, California, December 2014 
- Motivation - Introduction

- Notation and mathematical preliminaries

- Average consensus in digraphs

- Ratio consensus

- Ratio consensus with time-delays

- Contribution: Ratio consensus with dynamically changing directed topologies and time-delays

- Examples

- Concluding remarks and future directions 
- Distributed systems conveniently captured by digraphs

(1) Components represented by vertices (nodes)

(2) Communication and sensing links represented by edges

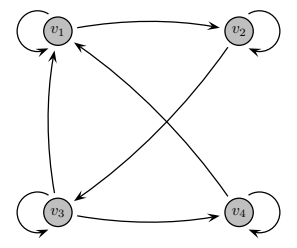

- Consider a network with nodes $\left(v_{1}, v_{2}, \ldots, v_{N}\right)$ (e.g., sensor networks, network of mappers in MapReduce, etc.)

- Nodes can receive information according to (possibly directed) communication links

- Each node $v_{j}$ has some initial value $x_{j}[0]$ (e.g., measurement, position, velocity, etc.) 


\section{Consensus and Average Consensus}

- Typical objective: Calculate functions of initial values in a distributed manner (e.g., $\max _{\ell}\left\{x_{\ell}[0]\right\}, \sum_{\ell} x_{\ell}^{2}[0]$, etc.)

- Consensus: All nodes calculate (in a distributed manner, each time using only local information) same function of initial values $x_{1}[0], x_{2}[0]$, $\ldots, x_{N}[0]$

- Average Consensus: All nodes calculate (in a distributed manner) the average $\bar{x} \equiv \frac{1}{N} \sum_{\ell=1}^{N} x_{\ell}[0]$ (where $N$ is the number of nodes)

- Possible centralized strategy: Route all values to a single entity (leading node) who then determines the function value (e.g., average) and routes it back to the nodes

- Average serves as primitive for estimation, inference and diagnosis (easily adjusted to arbitrary linear functions) 


\section{Graph Notation}

- $\operatorname{Digraph} \mathcal{G}=(\mathcal{V}, \mathcal{E})$

- Nodes (system components) $\mathcal{V}=\left\{v_{1}, v_{2}, \ldots, v_{N}\right\}$

- Edges (directed communication links) $\mathcal{E} \subseteq \mathcal{V} \times \mathcal{V}$ where $\left(v_{j}, v_{i}\right) \in \mathcal{E}$ iff node $v_{j}$ can receive information from node $v_{i}$

- In-neighbors $\mathcal{N}_{j}^{-}=\left\{v_{i} \mid\left(v_{j}, v_{i}\right) \in \mathcal{E}\right\}$; in-degree $\mathcal{D}_{j}^{-}=\left|\mathcal{N}_{j}^{-}\right|$

- Out-neighbors $\mathcal{N}_{j}^{+}=\left\{v_{l} \mid\left(v_{l}, v_{j}\right) \in \mathcal{E}\right\}$; out-degree $\mathcal{D}_{j}^{+}=\left|\mathcal{N}_{j}^{+}\right|$

- Adjacency matrix $A: A(j, i)=1$ if $\left(v_{j}, v_{i}\right) \in \mathcal{E} ; A(j, i)=0$ otherwise

- Undirected graph: $\left(v_{j}, v_{i}\right) \in \mathcal{E}$ iff $\left(v_{i}, v_{j}\right) \in \mathcal{E}$ (bidirectional links) In undirected graphs, we have (for each node $j$ ) $\mathcal{N}_{j}^{+}=\mathcal{N}_{j}^{-}$and $\mathcal{D}_{j}^{+}=\mathcal{D}_{j}^{-}=\mathcal{D}_{j} ;$ also, $A=A^{T}$

- (Strongly) connected (di)graph if for any $i, j \in \mathcal{V}, j \neq i$, there exists a (directed) path connecting them, i.e.,

$$
v_{i}=v_{i_{0}} \rightarrow v_{i_{1}}, v_{i_{1}} \rightarrow v_{i_{2}}, \ldots, v_{i_{t-1}} \rightarrow v_{i_{t}}=v_{j}
$$




\section{Conditions for Reaching Average Consensus}

\section{Linear Iterations}

- Average Consensus: All nodes calculate (in a distributed manner) the average $\frac{1}{N} \sum_{\ell=1}^{N} x_{\ell}[0]$ of initial values

- Consider linear iterations of the form

$$
x_{j}[k+1]=p_{j j} x_{j}[k]+\sum_{i \in \mathcal{N}_{j}^{-}} p_{j i} x_{i}[k], \forall j \in\{1,2, \ldots, N\}
$$

where $p_{j i}$ are constant weights and $x_{j}[0]$ is the initial value of node $j$

- Can be written in compact form as

$$
\begin{aligned}
x[k+1] & =P x[k] \\
x[0] & =\left[x_{1}[0] x_{2}[0] \ldots x_{N}[0]\right]^{T}
\end{aligned}
$$

- Weight matrix $P$ such that $P(j, i)=p_{j i}$

Note: $P(j, i)=0$ if $(j, i) \notin \mathcal{E}$ 


\section{Conditions for Asymptotic Average Consensus}

- Necessary and sufficient conditions on $P$ for asymptotic average consensus [Xiao \& Boyd, 2004]

(1) $P$ has a simple eigenvalue at 1 with left eigenvector $\mathbf{1}^{T}=\left[\begin{array}{llll}1 & 1 & \ldots & 1\end{array}\right]$ and right eigenvector $\mathbf{1}=\left[\begin{array}{llll}1 & 1 & \ldots & 1\end{array}\right]^{T}$

(2) All other eigenvalues of $P$ have magnitude strictly smaller than 1

- As $k \rightarrow \infty, P^{k} \rightarrow \frac{1}{N} 11^{T}$ which implies that

$$
\lim _{k \rightarrow \infty} x[k]=\frac{1}{N} \mathbf{1 1}^{T} x[0]=\left(\frac{\sum_{\ell=1}^{N} x_{\ell}[0]}{N}\right) \mathbf{1} \equiv \bar{x} \mathbf{1}
$$

- Nonnegative $p_{j i} \Longrightarrow P$ is primitive bistochastic

How to distributively reach the average in digraphs? 


\section{Average Consensus in Digraphs}

- In undirected graphs nodes have a number of ways to distributively choose their weights so as to form a bistochastic matrix

- Digraphs not as easy to handle, even in a centralized manner (because in general $\mathcal{D}_{j}^{+} \neq \mathcal{D}_{j}^{-}$)

- Approaches:

- Distributed algorithms to obtain weights that form bistochastic matrices [Gharesifard \& Cortés, 2012], [Rikos et al, 2014]

- Distributed approaches that introduce additional state variables and use broadcast gossip [Franceschelli et al, 2011], [Cai \& Ishii, 2011] to reach average consensus asymptotically

- Run two coupled iterations simultaneously (ratio consensus) [Benezit et al, 2010], [A.D. Domíngez-García \& C.N.H., 2010] that reach the average consensus asymptotically 


\section{Average Consensus using Ratio Consensus}

\section{Pair of Simultaneous Linear Iterations}

- Run two iterations [Benezit et al, 2010], [A.D. Domíngez-García \& C.N.H., 2010]

$$
\begin{array}{rl|rl}
\pi[k+1] & =P_{c} \pi[k] & x[k+1] & =P_{c} x[k] \\
\pi[0] & =\left[\pi_{1}[0] \ldots \pi_{N}[0]\right]^{T} & x[0] & =\mathbf{1}
\end{array}
$$

- Matrix $P_{c}$ st $P_{c}(l, j)=\frac{1}{1+\mathcal{D}_{j}^{+}}$for $v_{l} \in \mathcal{N}_{j}^{+}$(zero otherwise)

- Since $P_{c}$ is primitive column stochastic, we know that as $k \rightarrow \infty$, $P_{c}^{k} \rightarrow \mathbf{v} \mathbf{1}^{\top}$ for a strictly positive vector $\mathbf{v}$ such that $\mathbf{v}=P_{c} \mathbf{v}(\mathbf{v}$ is normalized so that its entries sum to unity)

- This implies that

$$
\begin{aligned}
& \lim _{k \rightarrow \infty} \pi[k]=\mathbf{v} 1^{T} \pi[0]=\left(\sum_{\ell=1}^{N} \pi_{\ell}[0]\right) \mathbf{v} \\
& \lim _{k \rightarrow \infty} x[k]=\mathbf{v} 1^{T} x[0]=N \mathbf{v}
\end{aligned}
$$

- For all nodes $j \in\{1,2, \ldots, N\}$, ratio converges

$$
\frac{\pi_{j}[k]}{x_{j}[k]} \rightarrow \frac{v_{j} \sum_{\ell=1}^{N} \pi_{\ell}[0]}{v_{j} N}=\frac{\sum_{\ell=1}^{N} \pi_{\ell}[0]}{N} \equiv \bar{\pi}
$$




\section{Average Consensus in the Presence of Delays}

- A transmission on link $\left(v_{j}, v_{i}\right)$ (from node $v_{i}$ to node $v_{j}$ ) at time-step $k$ can be delayed by $0,1, \ldots, D$ steps (arriving at node $v_{j}$ respectively at time-step $k+1, k+2, \ldots, k+D+1)$

- Unknown time-dependent delay function for each link $\left(v_{j}, v_{i}\right)$

$$
\operatorname{delay}_{(j, i)}[k]=d, \quad d \in\{0,1,2, \ldots, D\}
$$

- $\operatorname{delay}_{(j, j)}[k]=0$ for all $k$ and $v_{j} \in \mathcal{V}$

(own value is always available)

- For convenience, we use indicator functions

$$
I_{k, j, i}(d)=\left\{\begin{array}{l}
1, \text { if } \operatorname{delay}_{(j, i)}[k]=d \\
0, \text { otherwise }
\end{array}\right.
$$

Can ratio consensus reach average consensus in the presence of time-varying delays? 


\section{Average Consensus in the Presence of Delays}

- A transmission on link $\left(v_{j}, v_{i}\right)$ (from node $v_{i}$ to node $v_{j}$ ) at time-step $k$ can be delayed by $0,1, \ldots, D$ steps (arriving at node $v_{j}$ respectively at time-step $k+1, k+2, \ldots, k+D+1)$

- Unknown time-dependent delay function for each link $\left(v_{j}, v_{i}\right)$

$$
\operatorname{delay}_{(j, i)}[k]=d, \quad d \in\{0,1,2, \ldots, D\}
$$

- $\operatorname{delay}_{(j, j)}[k]=0$ for all $k$ and $v_{j} \in \mathcal{V}$

(own value is always available)

- For convenience, we use indicator functions

$$
I_{k, j, i}(d)=\left\{\begin{array}{l}
1, \text { if } \operatorname{delay}_{(j, i)}[k]=d \\
0, \text { otherwise }
\end{array}\right.
$$

Can ratio consensus reach average consensus in the presence of time-varying delays?

- Ratio consensus can be adopted to work in the presence of bounded time-varying delays [C.N.H. \& T.C., 2011], [C.N.H. \& T.C., 2014] 


\section{Average Consensus in the Presence of Delays}

\section{Running Two Delayed Iterations}

- Run two delayed iterations [C.N.H. \& T.C., 2011], [C.N.H. \& T.C., 2014]

$$
\begin{aligned}
\pi_{j}[k+1] & =p_{j j} \pi_{j}[k]+\sum_{i \in \mathcal{N}_{j}^{-}} p_{j i} \sum_{d=0}^{D} \pi_{i}[k-d] I_{k-d, j, i}(d) \\
\pi[0] & =\left[\pi_{1}[0] \ldots \pi_{N}[0]\right]^{T} \\
x_{j}[k+1] & =p_{j j} x_{j}[k]+\sum_{i \in \mathcal{N}_{j}^{-}} p_{j i} \sum_{d=0}^{D} x_{i}[k-d] I_{k-d, j, i}(d) \\
x[0] & =\mathbf{1}
\end{aligned}
$$

- Transmissions from node $v_{i}$ to node $v_{j}$ at time step $k$ undergo identical delays in both iterations (e.g., the pair of values are put in the same packet)

- The ratio $\pi_{j}[k] / x_{j}[k]$ converges in the limit to $\frac{\sum_{\ell=1}^{N} \pi_{\ell}[0]}{N}$ for all nodes $v_{j} \in \mathcal{V}$

Can ratio consensus also handle time-varying topologies on top of delays? 


\section{Average Consensus in the Presence of Delays and Switchings}

- Assumptions:

(1) At each time $k$, each node $v_{j}$ knows the number of nodes receiving its message - this assumption can be relaxed

(2) The delays are bounded

(3) There exist paths between any pair of nodes infinitely often

- Run two delayed iterations with time-varying coefficients

$$
\begin{aligned}
\pi_{j}[k+1] & =p_{j j}[k] \pi_{j}[k]+\sum_{d=0}^{D} \sum_{v_{i} \in \mathcal{N}_{j}^{-}[k-d]} \pi_{j \leftarrow i}[k-d] I_{k-d, j i}[d] \\
\pi[0] & =\left[\pi_{1}[0] \ldots \pi_{N}[0]\right]^{T} \\
x_{j}[k+1] & =p_{j j}[k] x_{j}[k]+\sum_{d=0}^{D} \sum_{v_{i} \in \mathcal{N}_{j}^{-}[k-d]} x_{j \leftarrow i}[k-d] l_{k-d, j i}[d] \\
x[0] & =1
\end{aligned}
$$

where $x_{j \leftarrow i}[k-d] \triangleq p_{j i}[k-d] x_{i}[k-d]$ is the value sent from node $v_{i}$ to node $v_{j}$ at time step $k-d$ that suffers delay $d$ 


\section{Example: 6-node digraph (1/2)}

- Initial conditions $\pi[0]=\left(\begin{array}{llllll}-1 & 1 & 2 & 3 & 4 & 3\end{array}\right)^{T}$ and $x[0]=\mathbf{1}$

- Each node $v_{j}$ chooses its self-weight and the weight of the links to its out-neighbors to be $\left(1+\mathcal{D}_{j}^{+}[k]\right)^{-1}$

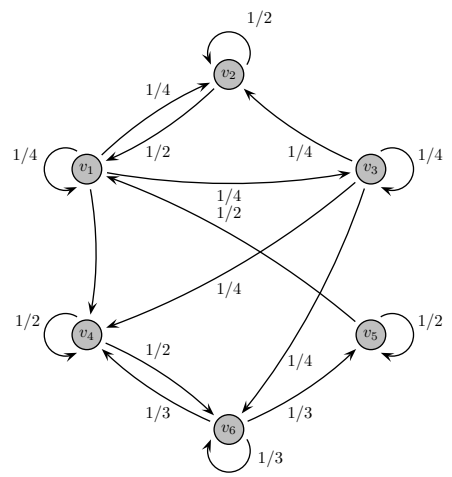

Connections and weights at time-instant $k_{1}$

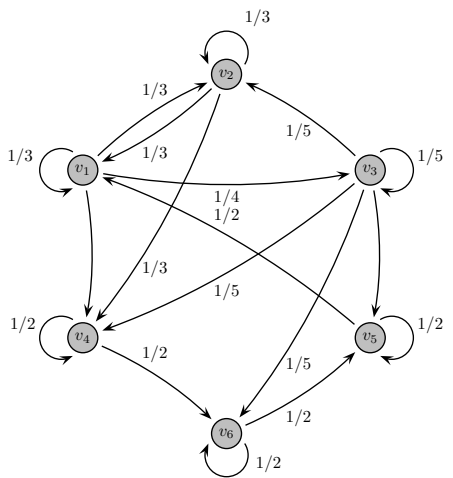

Connections and weights at time-instant $k_{2}$ 


\section{Examples}

\section{Example: 6-node digraph (2/2)}

- Iterations converge to the average

- maximum delay $\bar{\tau}=5$

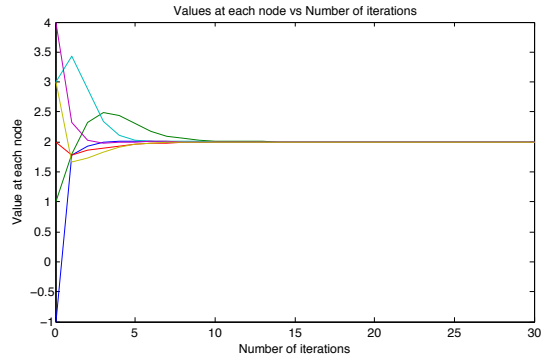

Switchings, no delays

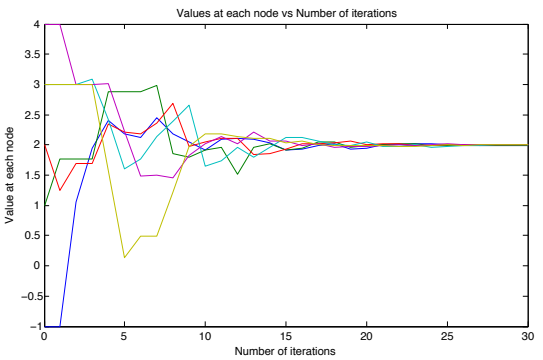

With switchings and delays 
- To handle delays (see [C.N.H. \& T.C., 2014]) we introduce DN "virtual" nodes (for a total of $(D+1) N$ nodes) so that we can write

$$
\bar{x}[k+1]=P[k] \bar{x}[k],
$$

where

$$
P[k] \triangleq\left(\begin{array}{ccccc}
P_{0}[k] & I_{N \times N} & 0 & \cdots & 0 \\
P_{1}[k] & 0 & I_{N \times N} & \cdots & 0 \\
\vdots & \vdots & \vdots & \ddots & \vdots \\
P_{D-1}[k] & 0 & 0 & \cdots & I_{N \times N} \\
P_{D}[k] & 0 & 0 & \cdots & 0
\end{array}\right)
$$

with

$$
\bar{x}[k]=\left(x^{T}[k] x^{(1)}[k] \ldots x^{(D)}[k]\right)^{T}, x^{(d)}[k]=\left(x_{1}^{(d)}[k] \ldots x_{N}^{(d)}[k]\right)
$$

- There exists $\ell$ and an infinite sequence of time instants $t_{0}, t_{1}, \ldots, t_{m}, \ldots$, such that for any $m \in \mathbb{Z}_{+}, 0<t_{m+1}-t_{m} \leq \ell<\infty, m \in \mathbb{Z}_{+}$, and the union of graphs $\mathcal{G}\left[t_{m}\right], \mathcal{G}\left[t_{m}+1\right], \ldots, \mathcal{G}\left[t_{m+1}-1\right]$ is strongly connected (assumption 3) 


\section{Sketch of proof (2/2)}

- Let $\bar{P}_{t_{m+1}-t_{m}} \triangleq P\left[t_{m+1}-1\right] P\left[t_{m+1}-2\right] \ldots P\left[t_{m}\right]$

- The union of graphs $\mathcal{G}\left[t_{m}\right], \mathcal{G}\left[t_{m}+1\right], \ldots, \mathcal{G}\left[t_{m+1}-1\right]$ is strongly connected and each matrix involved in the product has strictly positive elements on the diagonal $\Rightarrow \bar{P}_{t_{m+1}-t_{m}}$ is Stochastic, Indecomposable and Aperiodic (SIA)

- Products of matrices of the form $\bar{P}_{t_{m+1}-t_{m}}$ are SIA

- Wolfowitz theorem: For any $\epsilon>0$, there exists a finite integer $\nu(\epsilon) \in \mathbb{N}$, such that a finite word $W$ given by the product of a collection of $\nu$ stochastic matrices of the form $\bar{P}_{t_{m+1}-t_{m}}$ has all of its columns approximately the same

- This implies that

$$
\begin{aligned}
& \lim _{k \rightarrow \infty} \pi[k]=\mathbf{v}[k] \mathbf{1}^{T} \pi[0]=\left(\sum_{\ell=1}^{N} \pi_{\ell}[0]\right) \mathbf{v}[k] \\
& \lim _{k \rightarrow \infty} x[k]=\mathbf{v}[k] \mathbf{1}^{T} x[0]=N \mathbf{v}[k]
\end{aligned}
$$

- Therefore,

$$
\lim _{k \rightarrow \infty} \frac{\pi_{j}[k]}{x_{j}[k]}=\frac{\left(\sum_{\ell=1}^{N} \pi_{\ell}[0]\right) \mathbf{v}_{j}[k]}{N \mathbf{v}_{j}[k]}=\frac{\sum_{\ell=1}^{N} \pi_{\ell}[0]}{N}
$$




\section{Concluding Remarks and Future Directions}

\section{Conclusions:}

- Proposed a distributed algorithm

- operates in directed networks

- has limited local information exchanges

- converges to the average in the presence of delays and switchings

\section{Future work:}

- Investigation of quantized average consensus in directed graphs with switching topologies and delays 
[Xiao \& Boyd, 2004] L. Xiao and S. Boyd, "Fast linear iterations for distributed averaging," Systems and Control Letters, vol. 53, no. 1, pp. 6-78, Sept. 2004.

橹

[Benezit et al, 2010] F. Benezit, V. Blondel, P. Thiran, J. Tsitsiklis, and M. Vetterli, "Weighted gossip: Distributed averaging using non-doubly stochastic matrices," in Proc. of International Symposium on Information Theory, pp. 1753-1757, 2010.

[A.D. Domínguez-García \& C.N.H., 2011] A. D. Domínguez-García and C. N. Hadjicostis, "Coordination and control of distributed energy resources for provision of ancillary services," in Proceedings of the First IEEE International Conference on Smart Grid Communications, Oct. 2010, pp. 537-542.

言

[C.N.H. \& T.C., 2011] C. N. Hadjicostis and T. Charalambous, "Asynchronous coordination of distributed energy resources for the provisioning of ancillary services," in Proc. of Allerton Conference on Communication, Control, and Computing, pp. 1500-1507, 2011. 


\section{References}

[Cai \& Ishii, 2011] K. Cai and H. Ishii, "Quantized consensus and averaging on gossip digraphs," IEEE Transactions on Automatic Control, vol. 56, no. 9, pp. 2087-2100, Sept. 2011.

圆

[Franceschelli et al, 2011] M.Franceschelli, A. Giua, and C. Seatzu, "Distributed averaging in sensor networks based on broadcast gossip algorithms," IEEE Special Issue On Cognitive Sensor Networks, vol. 3, no. 3, pp. 808-817, 2011.

[Geresifard \& Cortés, 2012] B. Gharesifard and J. Cortés, "Distributed strategies for generating weight-balanced and doubly stochastic digraphs," European Journal of Control, vol. 18, no. 6, pp. 539-557, 2012.

[C.N.H. \& T.C., 2014] C. N. Hadjicostis and T. Charalambous, "Average Consensus in the Presence of Delays in Directed Graph Topologies", IEEE Transactions on Automatic Control, Volume 59 (3), pp.763-768, March 2014.

[Vaidya et al, 2012] N. H. Vaidya, C. N. Hadjicostis, and A. D.

Domínguez-García, "Robust average consensus over packet dropping links: Analysis via coefficients of ergodicity," in IEEE Conference on Decision and Control (CDC), Dec. 2012, pp. 2761-2766. 


\title{
For more information:
}

\author{
themiscekth.se \\ chadjic@ucy.ac.cy
}

\section{Questions?}




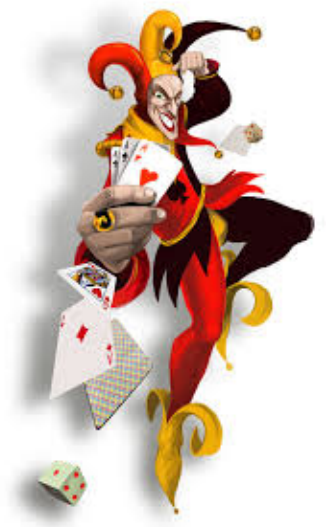

\author{
Joker slides
}


- Max number of time-slots required to receive acknowledgement is 2

- Node $v_{j}$ directs the weighted messages of the links that no longer exist as delayed information to itself
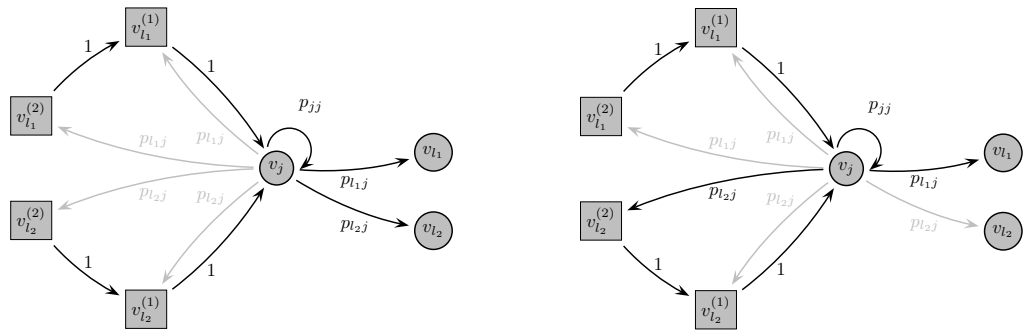
What if a node leave the network?

By an example (2/2)

Acknowledgement can be:

- via a distress signal

- via a path that reaches $v_{j}$

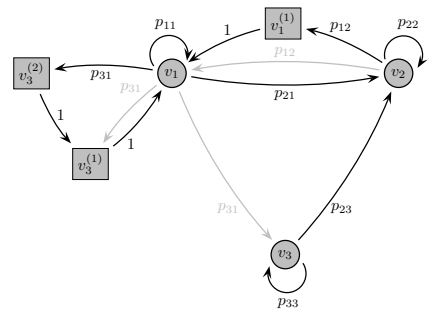


What if a node leave the network?

By an example (2/2)

Acknowledgement can be:

- via a distress signal

- via a path that reaches $v_{j}$

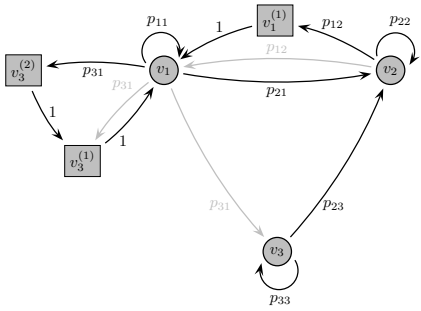

What if communication is not officially terminated? 


\section{What if a node leave the network?}

By an example (2/2)

\section{Acknowledgement can be:}

- via a distress signal

- via a path that reaches $v_{j}$

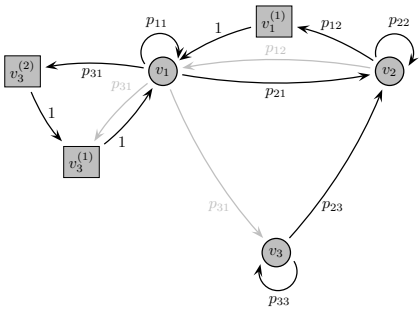

What if communication is not officially terminated?

- Each node $v_{j}$ broadcasts its own state, as well as the sum of all the values, called the total mass in [Vaidya et al, 2012] 